\title{
New Exact Solutions of Some Nonlinear Systems of Partial Differential Equations Using the First Integral Method
}

\author{
Shoukry Ibrahim Atia El-Ganaini ${ }^{1,2}$ \\ ${ }^{1}$ Mathematics Department, Faculty of Science, Damanhour University, Bahira 22514, Egypt \\ ${ }^{2}$ Mathematics Department, Faculty of Science and Humanity Studies at Al-Quwaiaiah, Shaqra University, \\ Al-Quwaiaiah 11971, Saudi Arabia \\ Correspondence should be addressed to Shoukry Ibrahim Atia El-Ganaini; ganaini5533@hotmail.com
}

Received 8 January 2013; Accepted 10 March 2013

Academic Editor: Elena Litsyn

Copyright (c) 2013 Shoukry Ibrahim Atia El-Ganaini. This is an open access article distributed under the Creative Commons Attribution License, which permits unrestricted use, distribution, and reproduction in any medium, provided the original work is properly cited.

The first integral method introduced by Feng is adopted for solving some important nonlinear systems of partial differential equations, including classical Drinfel'd-Sokolov-Wilson system (DSWE), (2+1)-dimensional Davey-Stewartson system, and generalized Hirota-Satsuma coupled KdV system. This method provides polynomial first integrals for autonomous planar systems. Through the established first integrals, exact traveling wave solutions are formally derived in a concise manner. This method can also be applied to nonintegrable equations as well as integrable ones.

\section{Introduction}

Over the four decades or so, nonlinear partial differential equations (NPDEs) have been the subject of extensive studies in various branches of nonlinear sciences.

A special class of analytical solutions, the so-called traveling waves, for NPDEs is of fundamental importance because lots of mathematical-physical models are often described by such wave phenomena.

Therefore, the investigation of traveling wave solutions is becoming more and more attractive in nonlinear sciences nowadays. However, not all equations posed of these models are solvable. As a result, many new techniques have been successfully developed by diverse groups of mathematicians and physicists, such as the Exp-function method [1-3], the sine-cosine method [4-6], the extended tanh-function method $[7,8]$, the modified extended tanh-function method [9-11], the F-expansion method [12], and the first integral method (or the algebraic curve method) [13]. Of these, the first integral method, which is based on the ring theory of commutative algebra, was first established by Feng [14-23].

This method was further developed by some other mathematicians [11, 24-33]. The method is reliable, effective, precise, and does not require complicated and tedious computations. The main idea of the first integral method is to find first integrals of nonlinear differential equations in polynomial form. Taking the polynomials with unknown polynomial coefficients into account, the method provides exact and explicit solutions. The interest in the present work is to implement the first integral method to stress its power in handling nonlinear partial differential equations, so that we can apply it for solving various types of these equations.

In Section 2, we describe this method for finding exact travelling wave solutions of nonlinear evolution equations. In Section 3, we illustrate this method in detail with the classical Drinfel'd-Sokolov-Wilson system (DSWE), the $(2+1)$ dimensional Davey-Stewartson system, and the generalized Hirota-Satsuma coupled KdV system. In Section 4, we give some conclusions.

\section{The First Integral Method}

Hosseini et al. in [30] have summarized the first integral method in the following steps.

Step 1. Consider the following nonlinear system of partial differential equations with independent variables $x$ and $t$ and dependent variables $u$ and $v$, 


$$
\begin{aligned}
& F_{1}\left(u, v, u_{t}, v_{t}, u_{x}, v_{x}, u_{t t}, v_{t t}, u_{x x}, v_{x x}, \ldots\right)=0, \\
& F_{2}\left(u, v, u_{t}, v_{t}, u_{x}, v_{x}, u_{t t}, v_{t t}, u_{x x}, v_{x x}, \ldots\right)=0 .
\end{aligned}
$$

Applying the transformations $u(x, t)=u(\xi)$ and $v(x, t)=$ $v(\xi)$, where $\xi=x-c t+\varsigma$, where $\varsigma$ is an arbitrary constant, converts (1) into a system of ordinary differential equations (ODEs)

$$
\begin{aligned}
& G_{1}\left(u, v, u^{\prime}, v^{\prime}, \ldots\right)=0, \\
& G_{2}\left(u, v, u^{\prime}, v^{\prime}, \ldots\right)=0,
\end{aligned}
$$

where the prime denotes the derivatives with respect to the same variable $\xi$.

Step 2. Using some mathematical operations, the system (2) is converted into a second-order ODE

$$
D\left(u, u^{\prime}, u^{\prime \prime}\right)=0 .
$$

Step 3. By introducing new variables $X=u(\xi)$ and $Y=u^{\prime}(\xi)$, (3) changes into a system of ODEs as the following system:

$$
\begin{gathered}
X^{\prime}=Y, \\
Y^{\prime}=H(X, Y) .
\end{gathered}
$$

Step 4. Now, the Division Theorem which is based on ring theory of commutative algebra is adopted to obtain one first integral to (4a) and (4b), which reduces (3) to a firstorder integrable ordinary differential equation. Finally, an exact solution to (1) is then established, through solving the resulting first-order integrable differential equation.

Let us now recall the Division Theorem for two variables in the complex domain $C(w, z)$.

Theorem 1 (Division Theorem). Suppose that $P(w, z)$, $Q(w, z)$ are polynomials in $C(w, z)$ and $P(w, z)$ is irreducible in $(w, z)$. If $Q(w, z)$ vanishes at all zero points of $(w, z)$, then there exists a polynomial $G(w, z)$ in $C(w, z)$ such that

$$
Q(w, z)=P(w, z) G(w, z) .
$$

The Division Theorem follows immediately from the HilbertNullstellensatz Theorem [34], but it can also be proved by using the complex analysis [35].

Theorem 2 (Hilbert-Nullstellensatz Theorem). Let $k$ be $a$ field and $L$ an algebraic closure of $k$.

(1) Every ideal $\gamma$ of $k\left[X_{1}, \ldots, X_{n}\right]$ not containing 1 admits at least one zero in $L^{n}$

(2) Let $x=\left(x_{1}, \ldots, x_{n}\right), y=\left(y_{1}, \ldots, y_{n}\right)$ be two elements of $L^{n}$; for the set of polynomials of $k\left[X_{1}, \ldots, X_{n}\right]$ zero at $x$ to be identical with the set of polynomials of $k\left[X_{1}, \ldots, X_{n}\right]$ zero at $y$, it is necessary and sufficient that there exists a $k$-automorphism $s$ of $L$ such that $y_{i}=s\left(x_{i}\right)$ for $1 \leq i \leq n$.
(3) For an ideal $\alpha$ of $k\left[X_{1}, \ldots, X_{n}\right]$ to be maximal, it is necessary and sufficient that there exists an $x$ in $L^{n}$ such that $\alpha$ is the set of polynomials of $k\left[X_{1}, \ldots, X_{n}\right]$ zero at $x$.

(4) For a polynomial $Q$ of $k\left[X_{1}, \ldots, X_{n}\right]$ to be zero on the set of zeros in $L^{n}$ of an ideal $\gamma$ of $\left[X_{1}, \ldots, X_{n}\right]$, it is necessary and sufficient that there exists an integer $m>0$ such that $Q^{m} \in \gamma$.

\section{Applications}

In this section, we investigate three NPDEs by using the first integral method.

3.1. Classical Drinfel'd-Sokolov-Wilson System. Consider the classical Drinfel'd-Sokolov-Wilson system [36]

$$
\begin{gathered}
u_{t}+p v v_{x}=0 \\
v_{t}+q v_{x x x}+r u v_{x}+s u_{x} v=0
\end{gathered}
$$

where $p, q, r, s$ are some nonzero parameters.

Recently, DSWE and the coupled DSWE, a special case of the classical DSWE, have been studied by several authors [36] and the references therein.

Using a complex variation $\eta$ defined as $\eta=k(x-c t)+\gamma$, we can convert (6) into ODEs, which read

$$
\begin{gathered}
-c u^{\prime}+p v v^{\prime}=0, \\
-c v^{\prime}+q k^{2} v^{\prime \prime \prime}+r u v^{\prime}+s u^{\prime} v=0
\end{gathered}
$$

where the prime denotes the derivative with respect to $\eta$.

Integrating (7), we obtain

$$
u=\frac{p v^{2}}{2 c}+c_{1}
$$

where $c_{1}$ is an arbitrary integration constant.

Substituting $u$ into (8) yields

$$
2 c q k^{2} v^{\prime \prime \prime}+p(r+2 s) v^{2} v^{\prime}+2 c\left(r c_{1}-c\right) v^{\prime}=0 .
$$

Integrating (10), we get

$$
2 c q k^{2} v^{\prime \prime}+p(r+2 s) \frac{v^{3}}{3}+2 c\left(r c_{1}-c\right) v=c_{2}
$$

where $c_{2}$ is an arbitrary integration constant.

By introducing new variables $X=v(\xi)$ and $Y=v^{\prime}(\xi),(11)$ changes into a system of ODEs

$$
\begin{gathered}
X^{\prime}=Y, \\
Y^{\prime}=\left(-\frac{p(r+2 s)}{6 c q k^{2}}\right) X^{3}-\left(\frac{r c_{1}-c}{q k^{2}}\right) X-\frac{c_{2}}{2 c q k^{2}} .
\end{gathered}
$$

According to the first integral method, we suppose that $X(\xi)$ and $Y(\xi)$ are nontrivial solutions of (12a) and (12b), and 
$P(X, Y)=\sum_{i=0}^{m} a_{i}(X) Y^{i}$ is an irreducible polynomial in the complex domain $C[X, Y]$ such that

$$
P[X(\xi), Y(\xi)]=\sum_{i=0}^{m} a_{i}(X(\xi)) Y^{i}(\xi)=0
$$

where $a_{i}(X),(i=0,1,2, \ldots, m)$ are polynomials of $X$ and $a_{m}(X) \neq 0$.

Equation (13) is called the first integral to (12a) and (12b). Due to the Division Theorem, there exists a polynomial $h(X)+g(X) Y$ in the complex domain $C[X, Y]$ such that

$$
\begin{aligned}
\frac{d P}{d \xi} & =\frac{\partial P}{\partial X} \frac{d X}{d \xi}+\frac{\partial P}{\partial Y} \frac{d Y}{d \xi} \\
& =[h(X)+g(X) Y] \sum_{i=0}^{m} a_{i}(X) Y^{i} .
\end{aligned}
$$

Here, we have considered one case only, assuming that $m=1$ in (13).

Suppose that $m=1$, by equating the coefficients of $Y^{i}$ $(i=2,1,0)$ on both sides of $(14)$, we have

$$
\begin{gathered}
a_{1}^{\prime}(X)=g(X) a_{1}(X), \\
a_{0}^{\prime}(X)=h(X) a_{1}(X)+g(X) a_{0}(X), \\
a_{1}(X)\left(\left(-\frac{p(r+2 s)}{6 c q k^{2}}\right) X^{3}-\left(\frac{r c_{1}-c}{q k^{2}}\right) X-\frac{c_{2}}{2 c q k^{2}}\right) \\
=h(X) a_{0}(X) .
\end{gathered}
$$

Since $a_{i}(X)(i=0,1)$ are polynomials, then from (15a) we have deduced that $a_{1}(X)$ is constant and $g(X)=0$. For simplicity, take $a_{1}(X)=1$.

Balancing the degrees of $h(X)$ and $a_{0}(X)$, we have concluded that $\operatorname{deg}(h(X))=1$ only. Suppose that $h(X)=$ $A X+B$, and $A \neq 0$, then we find $a_{0}(X)$

$$
a_{0}(X)=\frac{A}{2} X^{2}+B X+D
$$

where $D$ is an arbitrary integration constant.

Substituting $a_{0}(X), a_{1}(X)$ and $h(X)$ for $(15 \mathrm{c})$ and setting all the coefficients of powers $X$ to be zero, then we obtain a system of nonlinear algebraic equations and by solving it, we have obtained

$$
\begin{gathered}
c_{2}=0, \quad c_{1}=\frac{3 c-(\sqrt{3} k D q \sqrt{-p(r+2 s)}) / \sqrt{c q}}{3 r}, \\
A=\frac{\sqrt{-p(r+2 s)}}{\sqrt{3} k \sqrt{c q}}, \quad B=0, \\
c_{2}=0, \quad c_{1}=\frac{3 c+(k D q \sqrt{-p(r+2 s)}) / \sqrt{3} \sqrt{c q}}{r}, \\
A=-\frac{\sqrt{-p(r+2 s)}}{\sqrt{3} k \sqrt{c q}}, \quad B=0 .
\end{gathered}
$$

Setting (17a) and (17b) in (13) leads to

$$
\begin{gathered}
Y(\eta)+\left(\frac{\sqrt{-p(r+2 s)}}{2 \sqrt{3} k \sqrt{c q}} X^{2}(\eta)+D\right)=0, \\
Y(\eta)+\left(-\frac{\sqrt{-p(r+2 s)}}{2 \sqrt{3} k \sqrt{c q}} X^{2}(\eta)+D\right)=0 .
\end{gathered}
$$

Combining (18) with (12a), a first-order ordinary differential equation is derived, then by solving this derived equation and considering $X=v(\xi)$ and $v(x, t)=v(\xi)$, we have obtained

$$
\begin{aligned}
v_{1}(x, t)= & i(-3)^{1 / 4} c(q)^{1 / 4} \sqrt{k} \sqrt{D} \\
& \times \tan \left[\left(\left(-\frac{1}{3}\right)^{1 / 4} \sqrt{D}(p)^{1 / 4}(r+2 s)^{1 / 4}\right.\right. \\
& \left.\times\left[(k(x-c t)+\gamma)-3 \sqrt{c q} k \xi_{0}\right]\right) \\
& \left.\times\left(c(q)^{1 / 4} \sqrt{k}\right)^{-1}\right] \\
& \times\left((p)^{3 / 4}(r+2 s)^{1 / 4}\right)^{-1}, \\
v_{2}(x, t)= & -3)^{1 / 4} c(q)^{1 / 4} \sqrt{k} \sqrt{D} \\
& \times \tan \left[\left((-1)^{3 / 4} \sqrt{D}(p)^{1 / 4}(r+2 s)^{1 / 4}\right.\right. \\
& \times\left((p)^{1 / 4}(r+2 s)^{1 / 4}\right)^{-1}, \\
& \left.\times\left[(k(x-c t)+\gamma)-3 \sqrt{c q} k \xi_{0}\right]\right) \\
& \left.\left.\times(3)^{1 / 4} c(q)^{1 / 4} \sqrt{k}\right)^{-1}\right]
\end{aligned}
$$

respectively, where $\xi_{0}$ is an arbitrary integration constant.

Also, by considering the solution $u$ given by the relations (9), we have obtained

$$
\begin{aligned}
& u_{1}(x, t)=\left(\frac{p}{2 c}\right) \\
& \times\left[i(-3)^{1 / 4} c(q)^{1 / 4} \sqrt{k} \sqrt{D}\right. \\
& \times \tan \left[\left(\left(-\frac{1}{3}\right)^{1 / 4} \sqrt{D}(p)^{1 / 4}(r+2 s)^{1 / 4}\right.\right. \\
& \left.\quad \times\left[(k(x-c t)+\gamma)-3 \sqrt{c q} k \xi_{0}\right]\right) \\
& \left.\times\left(c(q)^{1 / 4} \sqrt{k}\right)^{-1}\right]
\end{aligned}
$$




$$
\begin{aligned}
& \left.\times\left((p)^{1 / 4}(r+2 s)^{1 / 4}\right)^{-1}\right]^{2} \\
& +\frac{3 c-(\sqrt{3} k D q \sqrt{-p(r+2 s)}) / \sqrt{c q}}{3 r},
\end{aligned}
$$

$$
\begin{aligned}
u_{2}(x, t)= & \left(\frac{p}{2 c}\right) \\
& \times\left[(-3)^{1 / 4} c(q)^{1 / 4} \sqrt{k} \sqrt{D}\right. \\
& \times \tan \left[\left((-1)^{3 / 4} \sqrt{D}(p)^{1 / 4}(r+2 s)^{1 / 4}\right.\right. \\
& \left.\times\left[(k(x-c t)+\gamma)-3 \sqrt{c q} k \xi_{0}\right]\right) \\
& \left.\times\left((p)^{1 / 4}(r+2 s)^{1 / 4}\right)^{-1}\right]^{2} \\
+ & \frac{3 c+(k D q \sqrt{-p(r+2 s)}) / \sqrt{3} \sqrt{c q}}{r},
\end{aligned}
$$

respectively, where $\xi_{0}$ is an arbitrary integration constant.

Thus, two solutions $\left(u_{1}, v_{1}\right)$ and $\left(u_{2}, v_{2}\right)$ have been obtained for the system (6).

Comparing these results with the results obtained in [36], it can be seen that the solutions here are new.

3.2. $(2+1)$-Dimensional Davey-Stewartson System. The $(2+$ 1)-dimensional Davey-Stewartson system [37] reads

$$
\begin{gathered}
i u_{t}+u_{x x}-u_{y y}-2|u|^{2} u-2 u v=0 \\
v_{x x}+v_{y y}+2\left(|u|^{2}\right)_{x x}=0 .
\end{gathered}
$$

This equation is completely integrable and used to describe the long-time evolution of a two-dimensional wave packet.

Using the wave variables

$$
\begin{gathered}
u=e^{i \theta} u(\xi), \quad v=v(\xi), \\
\theta=p x+q y+r t+\varepsilon, \quad \xi=k x+c y+d t+\gamma,
\end{gathered}
$$

where $p, q, r, k, c$, and $d$ are real constants, converts (23) into the ODE

$$
\begin{gathered}
\left(q^{2}-p^{2}-r\right) u+\left(k^{2}-c^{2}\right) u^{\prime \prime}-2 u^{3}-2 u v=0, \\
\left(k^{2}+c^{2}\right) v^{\prime \prime}+\left(u^{2}\right)^{\prime \prime}=0 .
\end{gathered}
$$

Integrating (26) in the system and neglecting constants of integration, we have found

$$
v=-\frac{u^{2}}{k^{2}+c^{2}} .
$$

Substituting (27) into (25) of the system and integrating we find

$$
\left(q^{2}-p^{2}-r\right) u+\left(k^{2}-c^{2}\right) u^{\prime \prime}-2 u^{3}+\frac{2 u^{3}}{k^{2}+c^{2}}=0 .
$$

By introducing new variables $X=u(\xi)$ and $Y=u^{\prime}(\xi),(28)$ changes into a system of ODEs

$$
\begin{gathered}
X^{\prime}=Y, \\
Y^{\prime}=\left(\frac{2-2 k^{2}-2 c^{2}}{c^{4}-k^{4}}\right) X^{3}+\left(\frac{q^{2}-p^{2}-r}{c^{2}-k^{2}}\right) X .
\end{gathered}
$$

According to the first integral method, we suppose that $X(\xi)$ and $Y(\xi)$ are nontrivial solutions of (29a) and (29b), and $P(X, Y)=\sum_{i=0}^{m} a_{i}(X) Y^{i}$ is an irreducible polynomial in the complex domain $C[X, Y]$ such that

$$
P[X(\xi), Y(\xi)]=\sum_{i=0}^{m} a_{i}(X(\xi)) Y^{i}(\xi)=0,
$$

where $a_{i}(X),(i=0,1,2, \ldots, m)$ are polynomials of $X$ and $a_{m}(X) \neq 0$.

Equation (30) is called the first integral to (29a) and (29b). Due to the Division Theorem, there exists a polynomial $h(X)+g(X) Y$ in the complex domain $C[X, Y]$ such that

$$
\begin{aligned}
\frac{d P}{d \xi} & =\frac{\partial P}{\partial X} \frac{d X}{d \xi}+\frac{\partial P}{\partial Y} \frac{d Y}{d \xi} \\
& =[h(X)+g(X) Y] \sum_{i=0}^{m} a_{i}(X) Y^{i} .
\end{aligned}
$$

Here, we have considered two different cases, assuming that $m=1$ and $m=2$ in (30).

Case 1. Suppose that $m=1$, by equating the coefficients of $Y^{i}(i=2,1,0)$ on both sides of $(31)$, we have

$$
\begin{gathered}
a_{1}^{\prime}(X)=g(X) a_{1}(X), \\
a_{0}^{\prime}(X)=h(X) a_{1}(X)+g(X) a_{0}(X), \\
a_{1}(X)\left(\left(\frac{2-2 k^{2}-2 c^{2}}{c^{4}-k^{4}}\right) X^{3}+\left(\frac{q^{2}-p^{2}-r}{c^{2}-k^{2}}\right) X\right) \\
=h(X) a_{0}(X) .
\end{gathered}
$$

Since $a_{i}(X)(i=0,1)$ are polynomials, then from (32a) it can be deduced that $a_{1}(X)$ is constant and $g(X)=0$. For simplicity, take $a_{1}(X)=1$.

Balancing the degrees of $h(X)$ and $a_{0}(X)$, it can be concluded that $\operatorname{deg}(h(X))=1$ only. Suppose that $h(X)=$ $A X+B$, and $A \neq 0$, then we find $a_{0}(X)$

$$
a_{0}(X)=\frac{A}{2} X^{2}+B X+D
$$

Substituting $a_{0}(X), a_{1}(X)$, and $h(X)$ for (32c) and setting all the coefficients of powers $X$ to be zero, then we obtain 
a system of nonlinear algebraic equations and by solving it, we obtain

$$
\begin{gathered}
D=\mp \frac{\sqrt{-\left(-1+c^{2}+k^{2}\right) /\left(c^{2}-k^{2}\right)} \sqrt{c^{2}+k^{2}}\left(p^{2}-q^{2}+r\right)}{2\left(-1+c^{2}+k^{2}\right)}, \\
B=0, \quad A=\mp \frac{2 \sqrt{-\left(-1+c^{2}+k^{2}\right) /\left(c^{2}-k^{2}\right)}}{\sqrt{c^{2}+k^{2}}} .
\end{gathered}
$$

Using the conditions (34) in (30), we obtain

$$
Y(\xi)= \pm \frac{\sqrt{-\left(-1+c^{2}+k^{2}\right) /\left(c^{2}-k^{2}\right)}}{\sqrt{c^{2}+k^{2}}} X^{2}(\xi) \pm D,
$$

respectively.

Combining (35) with (29a), we obtain the exact solutions to (28), and considering the solution $v$ given by the relation (27), thus the exact traveling wave solutions to the $(2+1)$ dimensional Davey-Stewartson system (23) were obtained and can be written as

$$
\begin{aligned}
u_{1,2}(x, y, t)= \pm \frac{i \sqrt{p^{2}-q^{2}+r}}{\sqrt{2-2 /\left(c^{2}+k^{2}\right)}} \\
\times \tanh \left[\sqrt{p^{2}-q^{2}+r}\right. \\
\times(k x+c y+d t+\gamma \\
\mp 2 i \sqrt{c-k} \sqrt{c+k} \\
\left.\times \sqrt{-1+c^{2}+k^{2}} \sqrt{c^{2}+k^{2}} \xi_{0}\right) \\
\left.\times(\sqrt{2} \sqrt{c-k} \sqrt{c+k})^{-1}\right] \\
\times \exp [i(p x+q y+r t+\varepsilon)]
\end{aligned}
$$$$
v_{1,2}(x, y, t)=\left(-\frac{1}{c^{2}+k^{2}}\right)
$$$$
\times\left[ \pm \frac{i \sqrt{p^{2}-q^{2}+r}}{\sqrt{2-2 /\left(c^{2}+k^{2}\right)}}\right.
$$$$
\times \tanh \left[\sqrt{p^{2}-q^{2}+r}\right.
$$$$
\times(k x+c y+d t+\gamma
$$$$
\mp 2 i \sqrt{c-k} \sqrt{c+k}
$$$$
\times \sqrt{-1+c^{2}+k^{2}}
$$$$
\left.\times \sqrt{c^{2}+k^{2}} \xi_{0}\right)
$$

$$
\left.\times(\sqrt{2} \sqrt{c-k} \sqrt{c+k})^{-1}\right]
$$

$$
\times \exp [i(p x+q y+r t+\varepsilon)]]^{2}
$$

respectively, where, $\xi_{0}$ is an arbitrary integration constant.

Case 2. Suppose that $m=2$, by equating the coefficients of $Y^{i}(i=3,2,1,0)$ on both sides of $(31)$, we have

$$
\begin{aligned}
& a_{2}^{\prime}(X)=g(X) a_{2}(X), \\
& a_{1}^{\prime}(X)=h(X) a_{2}(X)+g(X) a_{1}(X), \\
& a_{0}^{\prime}(X)+2 a_{2}(X)\left[\left(\frac{2-2 k^{2}-2 c^{2}}{c^{4}-k^{4}}\right) X^{3}\right. \\
& \left.\quad+\left(\frac{q^{2}-p^{2}-r}{c^{2}-k^{2}}\right) X\right] \\
& =h(X) a_{1}(X)+g(X) a_{0}(X), \\
& a_{1}(X)\left[\left(\frac{2-2 k^{2}-2 c^{2}}{c^{4}-k^{4}}\right) X^{3}+\left(\frac{q^{2}-p^{2}-r}{c^{2}-k^{2}}\right) X\right] \\
& =h(X) a_{0}(X) .
\end{aligned}
$$

Since, $a_{i}(X)(i=0,1,2)$ are polynomials, then from (38a) it can be deduced that $a_{2}(X)$ is a constant and $g(X)=0$. For simplicity, we take $a_{2}(X)=1$. Balancing the degrees of $h(X)$ and $a_{0}(X)$ it can be concluded that $\operatorname{deg}(h(X))=1$ only.

In this case, it was assumed that $h(X)=A X+B$, and $A \neq 0$, then we find $a_{1}(X)$ and $a_{0}(X)$ as follows:

$$
\begin{aligned}
a_{1}(X)= & \left(\frac{A}{2}\right) X^{2}+B X+D \\
a_{0}(X)= & \left(\frac{A^{2}}{8}-\frac{1-k^{2}-c^{2}}{c^{4}-k^{4}}\right) X^{4}+\frac{A B}{2} X^{3} \\
& +\left(\frac{A D+B^{2}}{2}-\frac{q^{2}-p^{2}-r}{c^{2}-k^{2}}\right) X^{2}+B D X+F
\end{aligned}
$$

where $A, B, D$, and $F$ are arbitrary constants.

Substituting $a_{0}(X), a_{1}(X), a_{2}(X)$, and $h(X)$ for $(38 \mathrm{~d})$ and setting all the coefficients of powers $X$ to be zero, a system of nonlinear algebraic equations was obtained and by solving it, we got

$$
F=-\frac{\left(c^{2}+k^{2}\right)\left(p^{2}-q^{2}+r\right)^{2}}{4\left(-c^{2}+c^{4}+k^{2}-k^{4}\right)}, \quad B=0,
$$




$$
\begin{gathered}
D=-\frac{p^{2}-q^{2}+r}{(c-k) \sqrt{c+k} \sqrt{-\left(-1+c^{2}+k^{2}\right) /(c-k)\left(c^{2}+k^{2}\right)}}, \\
A=\frac{4 \sqrt{-\left(-1+c^{2}+k^{2}\right) /(c-k)\left(c^{2}+k^{2}\right)}}{\sqrt{c+k}}, \\
D=\frac{\left(c^{2}+k^{2}\right)\left(p^{2}-q^{2}+r\right)^{2}}{4\left(-c^{2}+c^{4}+k^{2}-k^{4}\right)}, \quad B=0, \\
(c-k) \sqrt{c+k} \sqrt{-\left(-1+c^{2}+k^{2}\right) /(c-k)\left(c^{2}+k^{2}\right)}
\end{gathered}
$$

Using the conditions (40a) and (40b) in (30), we obtain

$Y(\xi)$

$$
\begin{aligned}
& =-\left[\sqrt{-\frac{-1+c^{2}+k^{2}}{(c-k)\left(c^{2}+k^{2}\right)}}\right. \\
& \times\left(\mp 4 \sqrt{(c+k)^{2}\left(-1+c^{2}+k^{2}\right)^{3}\left(c^{2}+k^{2}\right)\left(p^{2}-q^{2}+r\right) X^{2}(\xi)}\right. \\
& +(c-k)\left(-1+c^{2}+k^{2}\right) \\
& \left.\left.\times\left(-2 X^{2}(\xi)+\left(c^{2}+k^{2}\right)\left(p^{2}-q^{2}+r+2 X^{2}(\xi)\right)\right)\right)\right] \\
& \times\left(2(c+k)^{3 / 2}\left(\left(-1+c^{2}+k^{2}\right)^{2}\right)\right)^{-1},
\end{aligned}
$$

$Y(\xi)$

$$
\begin{aligned}
& =-\left[\sqrt{-\frac{-1+c^{2}+k^{2}}{(c-k)\left(c^{2}+k^{2}\right)}}\right. \\
& \times\left(\mp 4 \sqrt{(c+k)^{2}\left(-1+c^{2}+k^{2}\right)^{3}\left(c^{2}+k^{2}\right)\left(p^{2}-q^{2}+r\right) X^{2}(\xi)}\right. \\
& \quad-(c-k)\left(-1+c^{2}+k^{2}\right) \\
& \left.\left.\quad \times\left(-2 X^{2}(\xi)+\left(c^{2}+k^{2}\right)\left(p^{2}-q^{2}+r+2 X^{2}(\xi)\right)\right)\right)\right]
\end{aligned}
$$$$
\times\left(2(c+k)^{3 / 2}\left(\left(-1+c^{2}+k^{2}\right)^{2}\right)\right)^{-1}
$$

Combining (41a) and (41b) with (29a) we have obtained the exact solutions to (28), and considering the solution $v$ given by the relation (27), thus the exact traveling wave solutions to the (2+1)-dimensional Davey-Stewartson system (23) can be written as

$$
\begin{aligned}
& u_{3,4}(x, y, t) \\
& \begin{array}{l}
=\frac{\sqrt{p^{2}-q^{2}+r}}{2 \sqrt{1-1 /\left(c^{2}+k^{2}\right)}} \\
\times( \pm 2 \mp \sqrt{2} \\
\times \tanh \left[\left(\sqrt{p^{2}-q^{2}+r}\right.\right. \\
\times\left(\mp i \sqrt{-1+c^{2}+k^{2}} \sqrt{c^{2}+k^{2}}\right. \\
\times(k x+c y+d t+\gamma) \\
\pm 2 \sqrt{c-k} \sqrt{c+k} \\
\left.\left.\times\left(-1+c^{2}+k^{2}\right)\left(c^{2}+k^{2}\right) \xi_{0}\right)\right) \\
\times\left((\sqrt{2} \sqrt{c-k} \sqrt{c+k}) \sqrt{-1+c^{2}+k^{2}}\right. \\
\left.\left.\left.\times \sqrt{c^{2}+k^{2}}\right)^{-1}\right)\right] \\
\times \exp [i(p x+q y+r t+\varepsilon)],
\end{array}
\end{aligned}
$$$$
v_{3,4}(x, y, t)
$$$$
=-\left(\frac{1}{c^{2}+k^{2}}\right)
$$$$
\times\left[\frac{\sqrt{p^{2}-q^{2}+r}}{2 \sqrt{1-1 /\left(c^{2}+k^{2}\right)}}\right.
$$$$
\times( \pm 2 \mp \sqrt{2}
$$$$
\times \tanh \left[\left(\sqrt{p^{2}-q^{2}+r}\right.\right.
$$$$
\times\left(\mp i \sqrt{-1+c^{2}+k^{2}} \sqrt{c^{2}+k^{2}}\right.
$$$$
\times(k x+c y+d t+\gamma)
$$$$
\pm 2 \sqrt{c-k} \sqrt{c+k}
$$$$
\left.\left.\times\left(-1+c^{2}+k^{2}\right)\left(c^{2}+k^{2}\right) \xi_{0}\right)\right)
$$ 


$$
\begin{gathered}
\times\left((\sqrt{2} \sqrt{c-k} \sqrt{c+k}) \sqrt{-1+c^{2}+k^{2}}\right. \\
\left.\left.\left.\times \sqrt{c^{2}+k^{2}}\right)^{-1}\right)\right]
\end{gathered}
$$$$
\times \exp [i(p x+q y+r t+\varepsilon)]]^{2}
$$

$u_{5,6}(x, y, t)$

$$
\begin{aligned}
& =\frac{\sqrt{p^{2}-q^{2}+r}}{2 \sqrt{1-1 /\left(c^{2}+k^{2}\right)}} \\
& \times(\mp 2 \pm \sqrt{2} \\
& \times \tanh \left[\left(\sqrt{p^{2}-q^{2}+r}\right.\right. \\
& \times\left(\mp i \sqrt{-1+c^{2}+k^{2}} \sqrt{c^{2}+k^{2}}\right. \\
& \times(k x+c y+d t+\gamma) \\
& \mp 2 \sqrt{c-k} \sqrt{c+k} \\
& \left.\left.\times\left(-1+c^{2}+k^{2}\right)\left(c^{2}+k^{2}\right) \xi_{0}\right)\right) \\
& \times\left((\sqrt{2} \sqrt{c-k} \sqrt{c+k}) \sqrt{-1+c^{2}+k^{2}}\right. \\
& \left.\left.\left.\times \sqrt{c^{2}+k^{2}}\right)^{-1}\right)\right]
\end{aligned}
$$$$
\times \exp [i(p x+q y+r t+\varepsilon)]
$$

$$
\begin{aligned}
& v_{5,6}(x, y, t) \\
&=-\left(\frac{1}{c^{2}+k^{2}}\right) \\
& \times {\left[\frac{\sqrt{p^{2}-q^{2}+r}}{2 \sqrt{1-1 /\left(c^{2}+k^{2}\right)}}\right.} \\
& \times(\mp 2 \pm \sqrt{2} \\
& \times \tanh \left[\left(\sqrt{p^{2}-q^{2}+r}\right.\right. \\
& \quad \times\left(\mp i \sqrt{-1+c^{2}+k^{2}} \sqrt{c^{2}+k^{2}}\right. \\
& \quad \times(k x+c y+d t+\gamma)
\end{aligned}
$$

$$
\begin{aligned}
& \mp 2 \sqrt{c-k} \sqrt{c+k} \\
& \left.\left.\times\left(-1+c^{2}+k^{2}\right)\left(c^{2}+k^{2}\right) \xi_{0}\right)\right) \\
& \times\left((\sqrt{2} \sqrt{c-k} \sqrt{c+k}) \sqrt{-1+c^{2}+k^{2}}\right. \\
& \left.\left.\left.\times \sqrt{c^{2}+k^{2}}\right)^{-1}\right)\right]
\end{aligned}
$$$$
\times \exp [i(p x+q y+r t+\varepsilon)]]^{2}
$$

respectively, where $\xi_{0}$ is an arbitrary integration constant.

Equations (36)-(37) and (42)-(45) are new types of exact traveling wave solutions to the $(2+1)$-dimensional DaveyStewartson system (23). It could not be obtained by the methods presented in [37].

3.3. Generalized Hirota-Satsuma Coupled KdV System. Consider the generalized Hirota-Satsuma coupled KdV system [38]

$$
\begin{gathered}
u_{t}=\frac{1}{4} u_{x x x}+3 u u_{x}+3\left(w-v^{2}\right)_{x} \\
v_{t}=-\frac{1}{2} v_{x x x}-3 u v_{x} \\
w_{t}=-\frac{1}{2} w_{x x x}-3 u w_{x} .
\end{gathered}
$$

When $w=0$, (46)-(48) reduce to be the well-known HirotaSatsuma coupled KdV system. We seek traveling wave solutions for (46)-(48) in the form

$$
\begin{gathered}
u(x, t)=u(\xi), \quad v(x, t)=v(\xi), \\
w(x, t)=w(\xi), \quad \xi=k(x-c t)+\varsigma,
\end{gathered}
$$

where $\varsigma$ is an arbitrary constant.

Substituting (49) into (46)-(47) yields an ODE

$$
\begin{gathered}
-c k u^{\prime}=\frac{1}{4} k^{3} u^{\prime \prime \prime}+3 k u u^{\prime}+3 k\left(w-v^{2}\right)^{\prime}, \\
-c k v^{\prime}=-\frac{1}{2} k^{3} v^{\prime \prime \prime}-3 k u v^{\prime}, \\
-c k w^{\prime}=-\frac{1}{2} k^{3} w^{\prime \prime \prime}-3 k u w^{\prime} .
\end{gathered}
$$

Let

$$
\begin{gathered}
u=\alpha v^{2}+\beta v+\gamma, \\
w=A_{0} v+B_{0},
\end{gathered}
$$


where $\alpha, \gamma, \beta, A_{0}$, and $B_{0}$ are constants [38]. Inserting (53) into (50) and (51) integrating once we know that (50) and (51) give rise to the same equation

$$
k^{2} v^{\prime \prime}=-2 \alpha v^{3}-3 \beta v^{2}+2(c-3 \gamma) v+c_{1},
$$

where $c_{1}$ is an integration constant. Integrating (54) once again we have

$$
k^{2} v^{\prime 2}=-\alpha v^{4}-2 \beta v^{3}+2(c-3 \gamma) v^{2}+2 c_{1} v+c_{2},
$$

where $c_{2}$ is an integration constant. By means of (53)-(55) we get

$$
\begin{aligned}
k^{2} u^{\prime \prime}= & 2 \alpha k^{2} v^{\prime 2}+k^{2}(2 \alpha v+\beta) v^{\prime \prime} \\
= & 2 \alpha\left[-\alpha v^{4}-2 \beta v^{3}+2(c-3 \gamma) v^{2}+2 c_{1} v+c_{2}\right] \\
& +(2 \alpha v+\beta)\left[-2 \alpha v^{3}-3 \beta v^{2}+2(c-3 \gamma) v+c_{1}\right] .
\end{aligned}
$$

Integrating (50) once we have

$$
\frac{1}{4} k^{2} u^{\prime \prime}+\frac{3}{2} u^{2}+c u+3\left(w-v^{2}\right)+c_{3}=0
$$

where $c_{3}$ is an integration constant. Inserting (53) and (56) into (57) gives

$$
\begin{gathered}
3 \alpha c-3 \alpha \gamma+\frac{3}{4} \beta^{2}-3=0, \\
\frac{1}{2}\left[\alpha c_{1}+\beta c+\gamma \beta\right]+A_{0}=0, \\
\frac{1}{4}\left[2 \alpha c_{2}+\beta c_{1}\right]+\frac{3}{2} \gamma^{2}+c \gamma+3 B_{0}+c_{3}=0 .
\end{gathered}
$$

Let

$$
\begin{gathered}
c_{1}=\frac{1}{2 \alpha^{2}}\left[\beta^{3}+2 c \alpha \beta-6 \alpha \beta \gamma\right], \\
v(\xi)=a P(\xi)-\frac{\beta}{2 \alpha} .
\end{gathered}
$$

Therefore from (58), we have

$$
k^{2} P^{\prime \prime}(\xi)-a\left(\frac{3 \beta^{2}}{2 \alpha}+2 c-6 \gamma\right) P(\xi)+2 \alpha a^{3} P^{3}(\xi)=0
$$

By introducing new variables $X=P(\xi)$ and $Y=P^{\prime}(\xi),(60)$ changes into a system of ODEs

$$
\begin{gathered}
X^{\prime}=Y, \\
Y^{\prime}=-\left(\frac{2 \alpha a^{3}}{k^{2}}\right) X^{3}+\frac{a}{k^{2}}\left(\frac{3 \beta^{2}}{2 \alpha}+2 c-6 \gamma\right) X .
\end{gathered}
$$

According to the first integral method, we suppose that $X(\xi)$ and $Y(\xi)$ are nontrivial solutions of (61a) and (61b), and
$P(X, Y)=\sum_{i=0}^{m} a_{i}(X) Y^{i}$ is an irreducible polynomial in the complex domain $C[X, Y]$ such that

$$
P[X(\xi), Y(\xi)]=\sum_{i=0}^{m} a_{i}(X(\xi)) Y^{i}(\xi)=0,
$$

where $a_{i}(X),(i=0,1,2, \ldots, m)$ are polynomials of $X$ and $a_{m}(X) \neq 0$.

Equation (62) is called the first integral to (61a) and (61b). Due to the Division Theorem, there exists a polynomial $h(X)+g(X) Y$ in the complex domain $C[X, Y]$ such that

$$
\begin{aligned}
\frac{d P}{d \xi} & =\frac{\partial P}{\partial X} \frac{d X}{d \xi}+\frac{\partial P}{\partial Y} \frac{d Y}{d \xi} \\
& =[h(X)+g(X) Y] \sum_{i=0}^{m} a_{i}(X) Y^{i}
\end{aligned}
$$

Here, we have considered two different cases, assuming that $m=1$ and $m=2$ in (62).

Case 1. Suppose that $m=1$, by equating the coefficients of $Y^{i}(i=2,1,0)$ on both sides of $(63)$, we have

$$
\begin{gathered}
a_{1}^{\prime}(X)=g(X) a_{1}(X), \\
a_{0}^{\prime}(X)=h(X) a_{1}(X)+g(X) a_{0}(X), \\
a_{1}(X)\left[-\left(\frac{2 \alpha a^{3}}{k^{2}}\right) X^{3}+\frac{a}{k^{2}}\left(\frac{3 \beta^{2}}{2 \alpha}+2 c-6 \gamma\right) X\right] \\
=h(X) a_{0}(X) .
\end{gathered}
$$

Since $a_{i}(X)(i=0,1)$ are polynomials, then from (64a) it was deduced that $a_{1}(X)$ is constant and $g(X)=0$. For simplicity, take $a_{1}(X)=1$.

Balancing the degrees of $h(X)$ and $a_{0}(X)$, it was concluded that $\operatorname{deg}(h(X))=1$ only. Suppose that $h(X)=A X+B$, and $A \neq 0$, then we find

$$
a_{0}(X)=\frac{A}{2} X^{2}+B X+D,
$$

where $D$ is an arbitrary integration constant.

Substituting $a_{0}(X), a_{1}(X)$, and $h(X)$ for (64c) and setting all the coefficients of powers $X$ to be zero, then we have obtained a system of nonlinear algebraic equations and by solving it, we obtain

$$
\begin{gathered}
A=\mp \frac{2 i a^{3 / 2} \sqrt{\alpha}}{k}, \quad B=0, \\
c=\frac{\mp 4 i \sqrt{a} D k \alpha^{3 / 2}-3 \beta^{2}+12 \alpha \gamma}{4 \alpha} .
\end{gathered}
$$

Using the conditions (66) in (62), we obtain

$$
Y(\xi)=\left( \pm \frac{i a^{3 / 2} \sqrt{\alpha}}{k}\right) X^{2}(\xi)-D,
$$

respectively. Combining (67) with (61a), the exact solutions to (60) were obtained, and considering the solutions given by 
the relation (53), then the exact traveling wave solutions to the generalized Hirota-Satsuma coupled KdN system (46)(48) are obtained and can be written as

$$
\begin{aligned}
& u_{1}(x, t)=\alpha(a[(1-i) \sqrt{D} \sqrt{k} \\
& \times \tanh \left(\left(\left(\frac{1}{2}+\frac{i}{2}\right) a^{3 / 2} \sqrt{D} \alpha^{1 / 4}\right.\right. \\
&\left.\times\left[-(k(x-c t)+\varsigma)+2 k \xi_{0}\right]\right) \\
&\left.\times(\sqrt{k})^{-1}\right) \\
&\left.\left.\times\left(a^{3 / 2} \alpha^{1 / 4}\right)^{-1}\right]-\frac{\beta}{2 \alpha}\right)^{2} \\
&+\beta(a[(1-i) \sqrt{D} \sqrt{k} \\
& \times \tanh \left(\left(\frac{1}{2}+\frac{i}{2}\right) a^{3 / 2} \sqrt{D} \alpha^{1 / 4}\right. \\
& \times[-(k(x-c t)+\varsigma) \\
&\left.\left.\left.+2 k \xi_{0}\right]\right)(\sqrt{k})^{-1}\right) \\
&\left.\left.\times\left(a^{3 / 2} \alpha^{1 / 4}\right)^{-1}\right]-\frac{\beta}{2 \alpha}\right)+\gamma
\end{aligned}
$$

$v_{1}(x, t)=a[(1-i) \sqrt{D} \sqrt{k}$

$$
\begin{aligned}
\times \tanh (( & \left(\frac{1}{2}+\frac{i}{2}\right) a^{3 / 2} \sqrt{D} \alpha^{1 / 4} \\
& \left.\times\left[-(k(x-c t)+\varsigma)+2 k \xi_{0}\right]\right) \\
\times & \left.\left.(\sqrt{k})^{-1}\right)\left(a^{3 / 2} \alpha^{1 / 4}\right)^{-1}\right]-\frac{\beta}{2 \alpha},
\end{aligned}
$$

$w_{1}(x, t)=A_{0}(a[(1-i) \sqrt{D} \sqrt{k}$

$$
\begin{aligned}
& \times \tanh (\left(\left(\frac{1}{2}+\frac{i}{2}\right) a^{3 / 2} \sqrt{D} \alpha^{1 / 4}\right. \\
& \times {\left.\left[-(k(x-c t)+\varsigma)+2 k \xi_{0}\right]\right) } \\
& \times\left.(\sqrt{k})^{-1}\right) \\
&\left.\left.\times\left(a^{3 / 2} \alpha^{1 / 4}\right)^{-1}\right]-\frac{\beta}{2 \alpha}\right)+B_{0},
\end{aligned}
$$

$u_{2}(x, t)=\alpha(a[(1-i) \sqrt{D} \sqrt{k}$

$$
\times \tan \left(\left(\left(\frac{1}{2}+\frac{i}{2}\right) a^{3 / 2} \sqrt{D} \alpha^{1 / 4}\right.\right.
$$$$
\left.\times\left[-(k(x-c t)+\varsigma)+2 k \xi_{0}\right]\right)
$$$$
\left.\times(\sqrt{k})^{-1}\right)
$$$$
\left.\left.\times\left(a^{3 / 2} \alpha^{1 / 4}\right)^{-1}\right]-\frac{\beta}{2 \alpha}\right)^{2}
$$$$
+\beta(a[(1-i) \sqrt{D} \sqrt{k}
$$$$
\times \tanh \left(\left(\left(\frac{1}{2}+\frac{i}{2}\right) a^{3 / 2} \sqrt{D} \alpha^{1 / 4}\right.\right.
$$$$
\left.\times\left[-(k(x-c t)+\varsigma)+2 k \xi_{0}\right]\right)
$$$$
\left.\times(\sqrt{k})^{-1}\right)
$$$$
\left.\left.\times\left(a^{3 / 2} \alpha^{1 / 4}\right)^{-1}\right]-\frac{\beta}{2 \alpha}\right)+\gamma,
$$

$$
v_{2}(x, t)=a[(1-i) \sqrt{D} \sqrt{k}
$$

$$
\times \tan \left(\left(\left(\frac{1}{2}+\frac{i}{2}\right) a^{3 / 2} \sqrt{D} \alpha^{1 / 4}\right.\right.
$$

$$
\left.\times\left[-(k(x-c t)+\varsigma)+2 k \xi_{0}\right]\right)
$$

$$
\left.\times(\sqrt{k})^{-1}\right)
$$

$$
\left.\times\left(a^{3 / 2} \alpha^{1 / 4}\right)^{-1}\right]-\frac{\beta}{2 \alpha},
$$

$w_{2}(x, t)=A_{0}(a[(1-i) \sqrt{D} \sqrt{k}$

$$
\begin{aligned}
& \times \tan (\left(\left(\frac{1}{2}+\frac{i}{2}\right) a^{3 / 2} \sqrt{D} \alpha^{1 / 4}\right. \\
& \times {\left.\left[-(k(x-c t)+\varsigma)+2 k \xi_{0}\right]\right) } \\
& \times\left.(\sqrt{k})^{-1}\right) \\
&\left.\left.\times\left(a^{3 / 2} \alpha^{1 / 4}\right)^{-1}\right]-\frac{\beta}{2 \alpha}\right)+B_{0},
\end{aligned}
$$

(70) 
Case 2. Suppose that $m=2$, by equating the coefficients of $Y^{i}(i=3,2,1,0)$ on both sides of $(63)$, we have

$$
\begin{gathered}
a_{2}^{\prime}(X)=g(X) a_{2}(X), \\
a_{1}^{\prime}(X)=h(X) a_{2}(X)+g(X) a_{1}(X), \quad(74 \mathrm{a}) \\
a_{0}^{\prime}(X)+2 a_{2}(X)\left[-\left(\frac{2 \alpha a^{3}}{k^{2}}\right) X^{3}+\frac{a}{k^{2}}\left(\frac{3 \beta^{2}}{2 \alpha}+2 c-6 \gamma\right) X\right] \\
=h(X) a_{1}(X)+g(X) a_{0}(X), \\
a_{1}(X)\left[-\left(\frac{2 \alpha a^{3}}{k^{2}}\right) X^{3}+\frac{a}{k^{2}}\left(\frac{3 \beta^{2}}{2 \alpha}+2 c-6 \gamma\right) X\right] \\
=h(X) a_{0}(X) .
\end{gathered}
$$

Since $a_{i}(X)(i=0,1,2)$ are polynomials, then from $(74 \mathrm{a})$ it can be deduced that $a_{2}(X)$ is a constant and $g(X)=0$. For simplicity, we take $a_{2}(X)=1$. Balancing the degrees of $h(X)$ and $a_{0}(X)$ it can be concluded that $\operatorname{deg}(h(X))=1$, only.

In this case, it was assumed that $h(X)=A X+B$, and $A \neq 0$, then we find $a_{1}(X)$ and $a_{0}(X)$ as follows:

$$
\begin{aligned}
& a_{1}(X)=\left(\frac{A}{2}\right) X^{2}+B X+D \\
& a_{0}(X)=\left(\frac{A^{2}}{8}+\frac{\alpha a^{3}}{k^{2}}\right) X^{4}+\frac{1}{2}(A B) X^{3} \\
&+\left(\frac{A D+B^{2}}{2}+\frac{-a}{k^{2}}\left(\frac{3 \beta^{2}}{2 \alpha}\right)+2 c-6 \gamma\right) X^{2} \\
&+B D X+F
\end{aligned}
$$

where $A, B, D$, and $F$ are arbitrary constants.

Substituting $a_{0}(X), a_{1}(X), a_{2}(X)$, and $h(X)$ for $(74 \mathrm{~d})$ and setting all the coefficients of powers $X$ to be zero, a system of nonlinear algebraic equations was obtained and by solving it, we got

$$
\begin{gathered}
F=0, \quad c=\frac{3\left(-\beta^{2}+4 \alpha \gamma\right)}{4 \alpha}, \quad B=0, \quad D=0, \\
A=-\frac{4 i a^{3 / 2} \sqrt{\alpha}}{k}, \\
F=0, \quad c=\frac{3\left(-\beta^{2}+4 \alpha \gamma\right)}{4 \alpha}, \quad B=0, \quad D=0, \\
A=\frac{4 i a^{3 / 2} \sqrt{\alpha}}{k} .
\end{gathered}
$$

Using the conditions (76a) in (62), we obtain

$$
Y(\xi)=\frac{i a^{3} k X^{2}(\xi) \sqrt{\alpha} \mp \sqrt{-a^{3}\left(-2+a^{3}\right) k^{2} X^{4}(\xi) \alpha}}{2 k^{2}},
$$

respectively. Combining (77) with (61a), the exact solutions to (60) were obtained, and considering the solutions given by the relation (53), then the exact traveling wave solutions to the generalized Hirota-Satsuma coupled KdN system (46)(48) are obtained and can be written as

$$
\begin{aligned}
& u_{3}(x, t)=\alpha(a[-(2 k) \\
& \times\left(i a^{3 / 2}\left(a^{3 / 2}-\sqrt{-2+a^{3}}\right)\right. \\
& \left.\left.\times \sqrt{\alpha}[k(x-c t)+\varsigma]+2 k \xi_{0}\right)^{-1}\right] \\
& \left.-\frac{\beta}{2 \alpha}\right)^{2} \\
& +\beta(a[-(2 k) \\
& \times\left(i a^{3 / 2}\left(a^{3 / 2}-\sqrt{-2+a^{3}}\right)\right. \\
& \left.\left.\times \sqrt{\alpha}[k(x-c t)+\varsigma]+2 k \xi_{0}\right)^{-1}\right] \\
& \left.-\frac{\beta}{2 \alpha}\right)+\gamma \\
& v_{3}(x, t)=a[-(2 k) \\
& \times\left(i a^{3 / 2}\left(a^{3 / 2}-\sqrt{-2+a^{3}}\right)\right. \\
& \left.\left.\times \sqrt{\alpha}[k(x-c t)+\varsigma]+2 k \xi_{0}\right)^{-1}\right] \\
& -\frac{\beta}{2 \alpha}
\end{aligned}
$$

$$
\begin{aligned}
& w_{3}(x, t)=A_{0}(a[-(2 k) \\
& \times\left(i a^{3 / 2}\left(a^{3 / 2}-\sqrt{-2+a^{3}}\right)\right. \\
&\left.\left.\times \sqrt{\alpha}[k(x-c t)+\varsigma]+2 k \xi_{0}\right)^{-1}\right] \\
&-\left.\frac{\beta}{2 \alpha}\right)+B_{0}, \\
& u_{4}(x, t)=\alpha(a[-(2 k) \\
& \times\left(i a^{3 / 2}\left(a^{3 / 2}+\sqrt{-2+a^{3}}\right)\right. \\
&\left.\left.\times \sqrt{\alpha}[k(x-c t)+\varsigma]+2 k \xi_{0}\right)^{-1}\right] \\
&\left.-\frac{\beta}{2 \alpha}\right)^{2}
\end{aligned}
$$




$$
\begin{aligned}
& +\beta(a[-(2 k) \\
& \times\left(i a^{3 / 2}\left(a^{3 / 2}+\sqrt{-2+a^{3}}\right)\right. \\
& \left.\left.\times \sqrt{\alpha}[k(x-c t)+\varsigma]+2 k \xi_{0}\right)^{-1}\right] \\
& \left.-\frac{\beta}{2 \alpha}\right)^{2}+\gamma \\
& v_{4}(x, t)=a[-(2 k) \\
& \times\left(i a^{3 / 2}\left(a^{3 / 2}+\sqrt{-2+a^{3}}\right)\right. \\
& \left.\left.\times \sqrt{\alpha}[k(x-c t)+\varsigma]+2 k \xi_{0}\right)^{-1}\right] \\
& -\frac{\beta}{2 \alpha} \\
& w_{4}(x, t)=A_{0}(a[-(2 k) \\
& \times\left(i a^{3 / 2}\left(a^{3 / 2}+\sqrt{-2+a^{3}}\right)\right. \\
& \left.\left.\times \sqrt{\alpha}[k(x-c t)+\varsigma]+2 k \xi_{0}\right)^{-1}\right] \\
& \left.-\frac{\beta}{2 \alpha}\right)+B_{0}
\end{aligned}
$$

Similarly, as for the case of (76b) from (62) we obtain

$$
Y(\xi)=\mp \frac{ \pm i a^{3} k X^{2}(\xi) \sqrt{\alpha}+\sqrt{-a^{3}\left(-2+a^{3}\right) k^{2} X^{4}(\xi) \alpha}}{2 k^{2}},
$$

respectively. Combining (84) with (61a), the exact solutions to (60) were obtained, and considering the solutions given by the relation (53), then the exact traveling wave solutions to the generalized Hirota-Satsuma coupled KdN system (46)(48) are obtainred and can be written as

$$
\begin{aligned}
u_{5}(x, t)=\alpha(a[ & -(2 k) \\
& \times\left(-i a^{3 / 2}\left(a^{3 / 2}+\sqrt{-2+a^{3}}\right)\right. \\
& \left.\left.\times \sqrt{\alpha}[k(x-c t)+\varsigma]+2 k \xi_{0}\right)^{-1}\right] \\
\left.-\frac{\beta}{2 \alpha}\right)^{2} & \\
+\beta(a[- & (2 k) \\
& \times\left(-i a^{3 / 2}\left(a^{3 / 2}+\sqrt{-2+a^{3}}\right)\right.
\end{aligned}
$$$$
\left.\left.\times \sqrt{\alpha}[k(x-c t)+\varsigma]+2 k \xi_{0}\right)^{-1}\right]
$$$$
\left.-\frac{\beta}{2 \alpha}\right)+\gamma
$$$$
v_{5}(x, t)=a[-
$$

$$
\begin{aligned}
\times( & -i a^{3 / 2}\left(a^{3 / 2}+\sqrt{-2+a^{3}}\right) \\
& \left.\left.\times \sqrt{\alpha}[k(x-c t)+\varsigma]+2 k \xi_{0}\right)^{-1}\right]-\frac{\beta}{2 \alpha},
\end{aligned}
$$

$$
\begin{aligned}
w_{5}(x, t)=A_{0}(a[ & -(2 k) \\
& \times\left(-i a^{3 / 2}\left(a^{3 / 2}+\sqrt{-2+a^{3}}\right)\right. \\
& \left.\left.\times \sqrt{\alpha}[k(x-c t)+\varsigma]+2 k \xi_{0}\right)^{-1}\right] \\
\left.-\frac{\beta}{2 \alpha}\right) & +B_{0},
\end{aligned}
$$$$
u_{6}(x, t)=\alpha(a[-(2 k)
$$$$
\times\left(i a^{3 / 2}\left(-a^{3 / 2}+\sqrt{-2+a^{3}}\right)\right.
$$$$
\left.\left.\times \sqrt{\alpha}[k(x-c t)+\varsigma]+2 k \xi_{0}\right)^{-1}\right]
$$$$
\left.-\frac{\beta}{2 \alpha}\right)^{2}
$$$$
+\beta(a[-(2 k)
$$$$
\times\left(i a^{3 / 2}\left(-a^{3 / 2}+\sqrt{-2+a^{3}}\right)\right.
$$$$
\left.\left.\times \sqrt{\alpha}[k(x-c t)+\varsigma]+2 k \xi_{0}\right)^{-1}\right]
$$

$$
\begin{gathered}
\left.-\frac{\beta}{2 \alpha}\right)+\gamma \\
v_{6}(x, t)=a[-(2 k) \\
\times\left(i a^{3 / 2}\left(-a^{3 / 2}+\sqrt{-2+a^{3}}\right)\right. \\
\left.\left.\quad \times \sqrt{\alpha}[k(x-c t)+\varsigma]+2 k \xi_{0}\right)^{-1}\right]-\frac{\beta}{2 \alpha},
\end{gathered}
$$




$$
\begin{aligned}
w_{6}(x, t)=A_{0}(a[ & -(2 k) \\
& \times\left(i a^{3 / 2}\left(-a^{3 / 2}+\sqrt{-2+a^{3}}\right)\right. \\
& \left.\left.\times \sqrt{\alpha}[k(x-c t)+\varsigma]+2 k \xi_{0}\right)^{-1}\right] \\
\left.-\frac{\beta}{2 \alpha}\right) & +B_{0},
\end{aligned}
$$

respectively, where $\xi_{0}$ is an arbitrary integration constant.

Comparing the results (68)-(73), (78)-(83), and (85)(90) with the results in $[38,39]$, it can be seen that the solutions here are new.

Remark 3. We note that our results were based on the assumptions $m=1$ and $m=2$. The discussion becomes more complicated for $m=3$ and $m=4$ because the hyper-elliptic integrals, the irregular singular point theory, and the elliptic integrals of the second kind are involved. Also, we do not need to consider the case $m \geq 5$ because an algebraic equation with its degree greater than or equal to 5 is generally not solvable.

\section{Conclusion}

In this paper, the first integral method was applied successfully to obtain solutions of some important nonlinear systems, namely, the classical Drinfel'd-Sokolov-Wilson system (DSWE), the $(2+1)$-dimensional Davey-Stewartson system, and the generalized Hirota-Satsuma coupled KdV system. Also, we conclude that the proposed method is powerful, effective, and can be extended to solve more other nonlinear evolution equations as well as linear ones, and this will be done in a future work.

\section{Acknowledgment}

The author would like to thank the referees for their useful comments which led to some improvements of the current paper.

\section{References}

[1] J.-H. He and X.-H. Wu, "Exp-function method for nonlinear wave equations," Chaos, Solitons \& Fractals, vol. 30, no. 3, pp. 700-708, 2006.

[2] J.-H. He and M. A. Abdou, "New periodic solutions for nonlinear evolution equations using Exp-function method," Chaos, Solitons \& Fractals, vol. 34, no. 5, pp. 1421-1429, 2007.

[3] A. Bekir and A. Boz, "Exact solutions for nonlinear evolution equations using Exp-function method," Physics Letters A, vol. 372, no. 10, pp. 1619-1625, 2008.

[4] A.-M. Wazwaz, "A sine-cosine method for handling nonlinear wave equations," Mathematical and Computer Modelling, vol. 40, no. 5-6, pp. 499-508, 2004.

[5] A.-M. Wazwaz, "Exact solutions for the ZK-MEW equation by using the tanh and sine-cosine methods," International Journal of Computer Mathematics, vol. 82, no. 6, pp. 699-708, 2005.
[6] E. Yusufoğlu and A. Bekir, "Solitons and periodic solutions of coupled nonlinear evolution equations by using the sine-cosine method," International Journal of Computer Mathematics, vol. 83, no. 12, pp. 915-924, 2006.

[7] E. Fan, "Extended tanh-function method and its applications to nonlinear equations," Physics Letters A, vol. 277, no. 4-5, pp. 212-218, 2000.

[8] E. Fan, "Traveling wave solutions for generalized HirotaSatsuma coupled KdV systems," Naturforsch A, vol. 56, pp. 312318, 2001.

[9] S. A. Elwakil, S. K. El-Labany, M. A. Zahran, and R. Sabry, "Modified extended tanh-function method and its applications to nonlinear equations," Applied Mathematics and Computation, vol. 161, no. 2, pp. 403-412, 2005.

[10] S. A. Elwakil, S. K. El-labany, M. A. Zahran, and R. Sabry, "Modified extended tanh-function method for solving nonlinear partial differential equations," Physics Letters A, vol. 299, no. 2-3, pp. 179-188, 2002.

[11] F. Taşcan and A. Bekir, "Travelling wave solutions of the Cahn-Allen equation by using first integral method," Applied Mathematics and Computation, vol. 207, no. 1, pp. 279-282, 2009.

[12] Z. Sheng, "The periodic wave solutions for the $(2+1)$ dimensional Konopelchenko-Dubrovsky equations," Chaos, Solitons \& Fractals, vol. 30, no. 5, pp. 1213-1220, 2006.

[13] B. Lu, H. Zhang, and F. Xie, "Travelling wave solutions of nonlinear partial equations by using the first integral method," Applied Mathematics and Computation, vol. 216, no. 4, pp. 13291336, 2010.

[14] Z. Feng, "On explicit exact solutions to the compound BurgersKdV equation," Physics Letters A, vol. 293, no. 1-2, pp. 57-66, 2002.

[15] Z. Feng, "Exact solution to an approximate sine-Gordon equation in $(n+1)$-dimensional space," Physics Letters A, vol. 302, no. 2-3, pp. 64-76, 2002.

[16] Z. Feng and X. Wang, "The first integral method to the two-dimensional Burgers-Korteweg-de Vries equation," Physics Letters A, vol. 308, no. 2-3, pp. 173-178, 2003.

[17] Z. Feng, "The first-integral method to study the BurgersKorteweg-de Vries equation," Journal of Physics. A. Mathematical and General, vol. 35, no. 2, pp. 343-349, 2002.

[18] Z. Feng and G. Chen, "Solitary wave solutions of the compound Burgers-Korteweg-de Vries equation," Physica A: Statistical Mechanics and Its Applications, vol. 352, no. 2-4, pp. 419-435, 2005.

[19] Z. Feng, "Traveling wave behavior for a generalized Fisher equation," Chaos, Solitons \& Fractals, vol. 38, no. 2, pp. 481-488, 2008.

[20] Z. Feng and Y. Li, "Complex traveling wave solutions to the Fisher equation," Physica A. Statistical Mechanics and Its Applications, vol. 366, pp. 115-123, 2006.

[21] Z. Feng, "Exact solution to an approximate sine-Gordon equation in $(n+1)$-dimensional space," Physics Letters A, vol. 302, no. 2-3, pp. 64-76, 2002.

[22] Z. Feng and X. Wang, "The first integral method to the two-dimensional Burgers-Korteweg-de Vries equation," Physics Letters A, vol. 308, no. 2-3, pp. 173-178, 2003.

[23] Z. Feng and R. Knobel, "Traveling waves to a BurgersKorteweg-de Vries-type equation with higher-order nonlinearities," Journal of Mathematical Analysis and Applications, vol. 328, no. 2, pp. 1435-1450, 2007. 
[24] F. Tascan, A. Bekir, and M. Koparan, "Travelling wave solutions of nonlinear evolution equations by using the first integral method," Communications in Nonlinear Science and Numerical Simulation, vol. 14, no. 5, pp. 1810-1815, 2009.

[25] H. Li and Y. Guo, "New exact solutions to the FitzHughNagumo equation," Applied Mathematics and Computation, vol. 180, no. 2, pp. 524-528, 2006.

[26] X. Deng, "Travelling wave solutions for the generalized BurgersHuxley equation," Applied Mathematics and Computation, vol. 204, no. 2, pp. 733-737, 2008.

[27] S. I. El-Ganaini, "Travelling wave solutions of the ZakharovKuznetsov equation in plasmas with power law nonlinearity," International Journal of Contemporary Mathematical Sciences, vol. 6, no. 45-48, pp. 2353-2366, 2011.

[28] S. I. A. El-Ganaini, “Travelling wave solutions to the generalized Pochhammer-Chree (PC) equations using the first integral method," Mathematical Problems in Engineering, vol. 2011, Article ID 629760, 13 pages, 2011.

[29] S. I. A. El-Ganaini, "The first integral method to the nonlinear Schrodinger equations in higher dimensions," Abstract and Applied Analysis, vol. 2013, Article ID 349173, 10 pages, 2013.

[30] K. Hosseini, R. Ansari, and P. Gholamin, "Exact solutions of some nonlinear systems of partial differential equations by using the first integral method," Journal of Mathematical Analysis and Applications, vol. 387, no. 2, pp. 807-814, 2012.

[31] K. R. Raslan, "The first integral method for solving some important nonlinear partial differential equations," Nonlinear Dynamics. An International Journal of Nonlinear Dynamics and Chaos in Engineering Systems, vol. 53, no. 4, pp. 281-286, 2008.

[32] S. Abbasbandy and A. Shirzadi, "The first integral method for modified Benjamin-Bona-Mahony equation," Communications in Nonlinear Science and Numerical Simulation, vol. 15, no. 7, pp. 1759-1764, 2010.

[33] I. Aslan, "Travelling wave solutions to nonlinear physical models by means of the first integral method," PramanaJournal of Physics, vol. 76, no. 4, pp. 533-542, 2011.

[34] N. Bourbaki, Commutative Algebra, Addison-Wesley, Paris, France, 1972.

[35] Z. Feng, "Algebraic curve solution for second-order polynomial autonomous systems," Electronic Journal of Linear Algebra, vol. 8, pp. 14-25, 2001.

[36] E. Misirli and Y. Gurefe, "Exact solutions of the Drinfel'dSokolov-Wilson equation using the Exp-function method," Applied Mathematics and Computation, vol. 216, no. 9, pp. 26232627, 2010.

[37] A. Bekir and A. C. Cevikel, "New solitons and periodic solutions for nonlinear physical models in mathematical physics," Nonlinear Analysis. Real World Applications, vol. 11, no. 4, pp. 3275-3285, 2010.

[38] S. A. El-Wakil and M. A. Abdou, "New exact travelling wave solutions using modified extended tanh-function method," Chaos, Solitons \& Fractals, vol. 31, no. 4, pp. 840-852, 2007.

[39] S. A. El-Wakil, M. A. Madkour, M. T. Attia, A. Elhanbaly, and M. A. Abdou, "Construction of periodic and solitary wave solutions for nonlinear evolution equations," International Journal of Nonlinear Science, vol. 7, no. 1, pp. 12-20, 2009. 


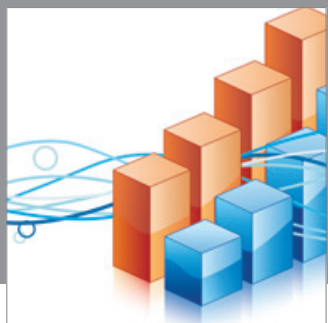

Advances in

Operations Research

mansans

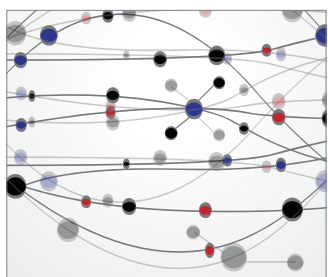

The Scientific World Journal
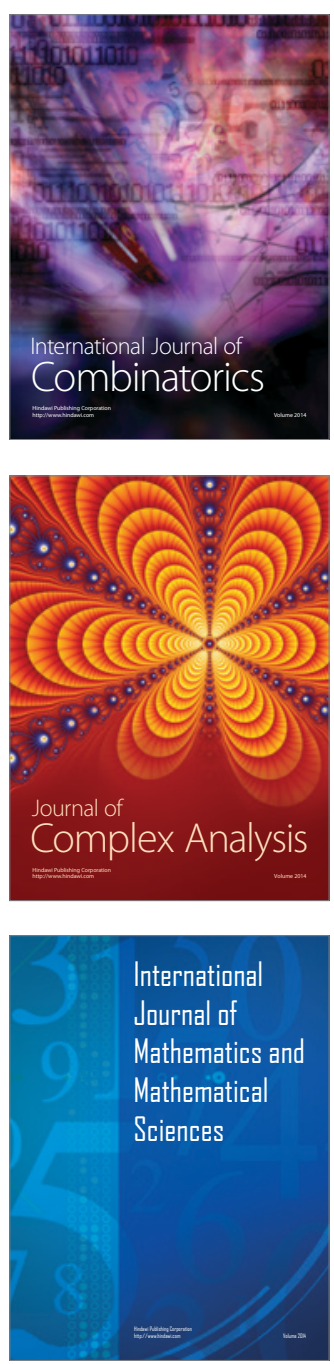
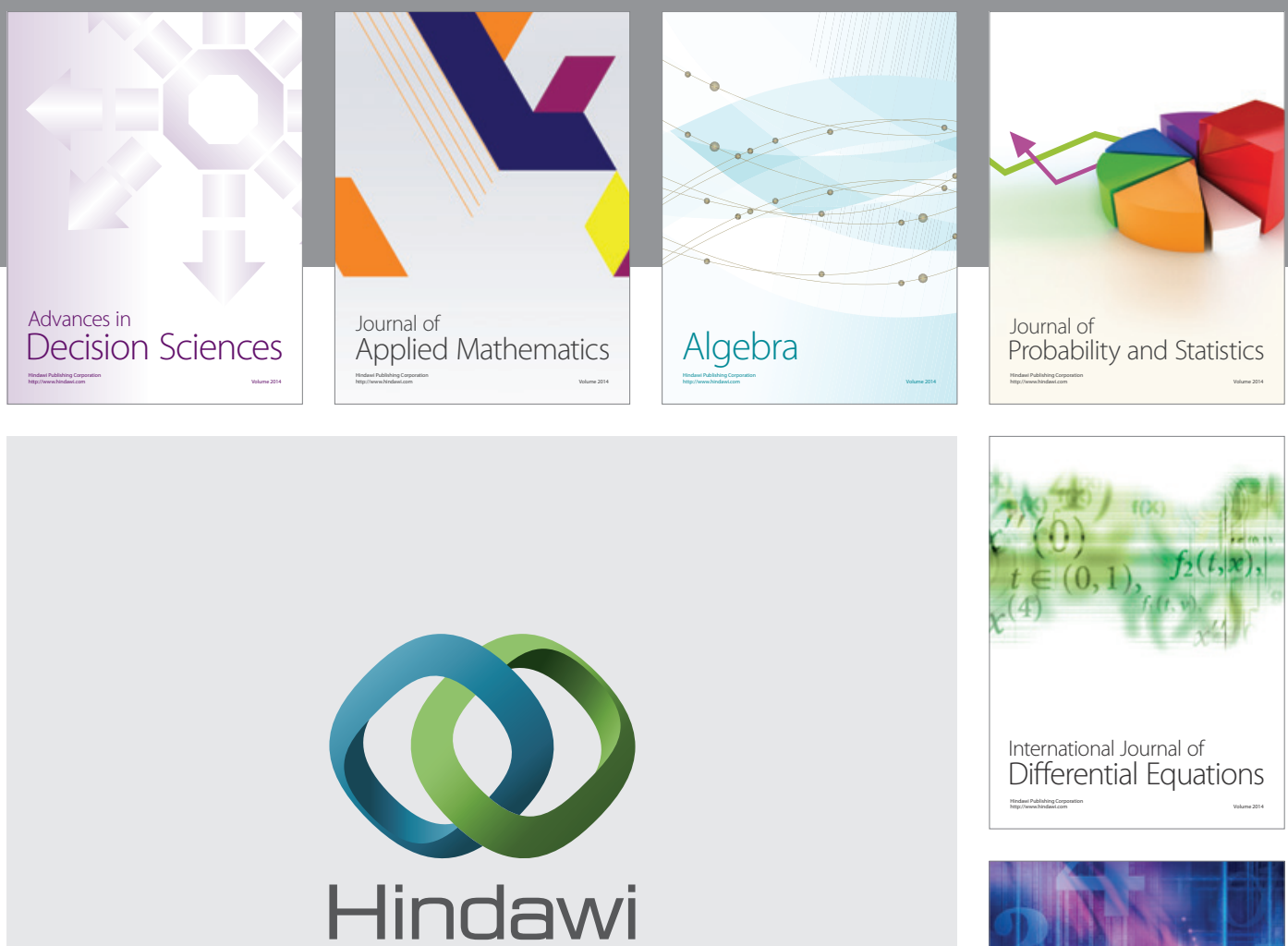

Submit your manuscripts at http://www.hindawi.com
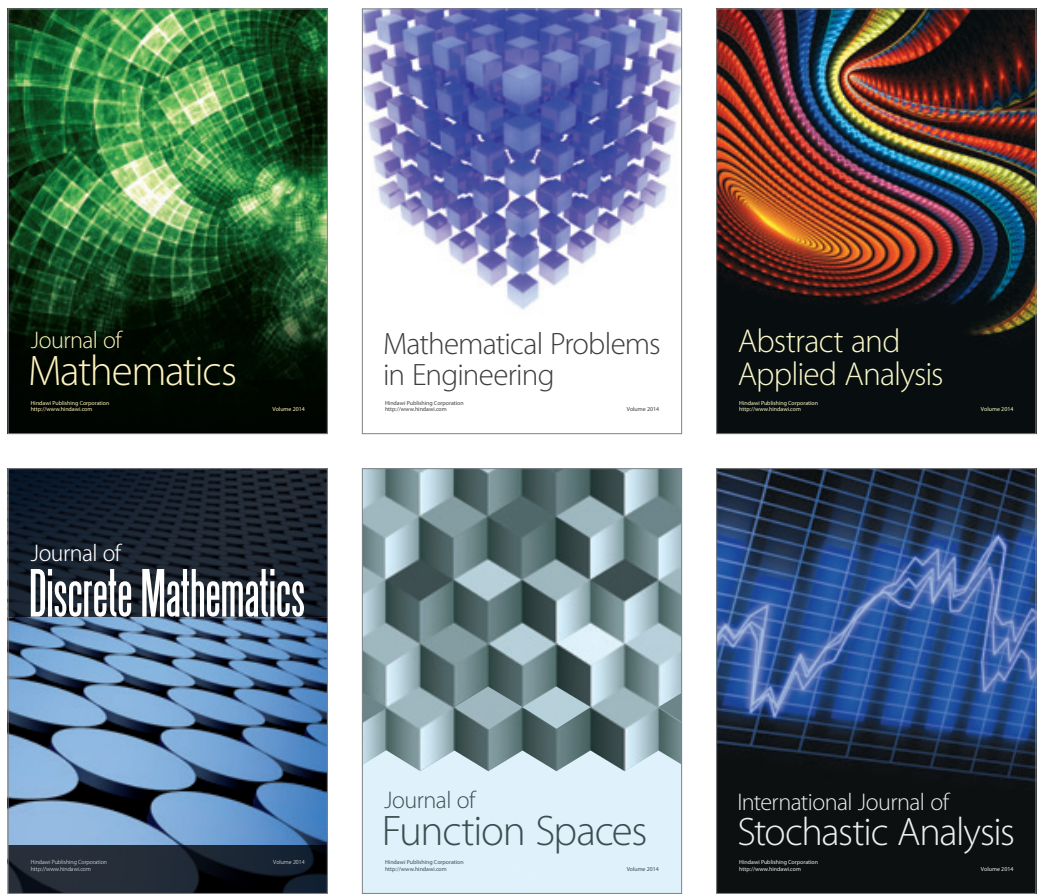

Journal of

Function Spaces

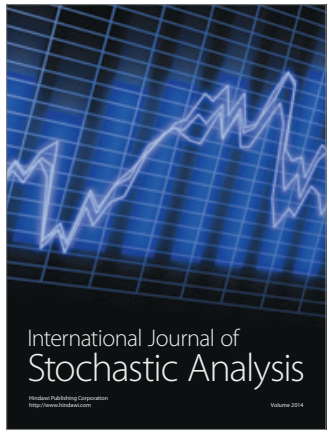

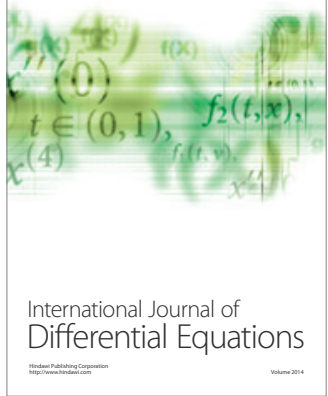
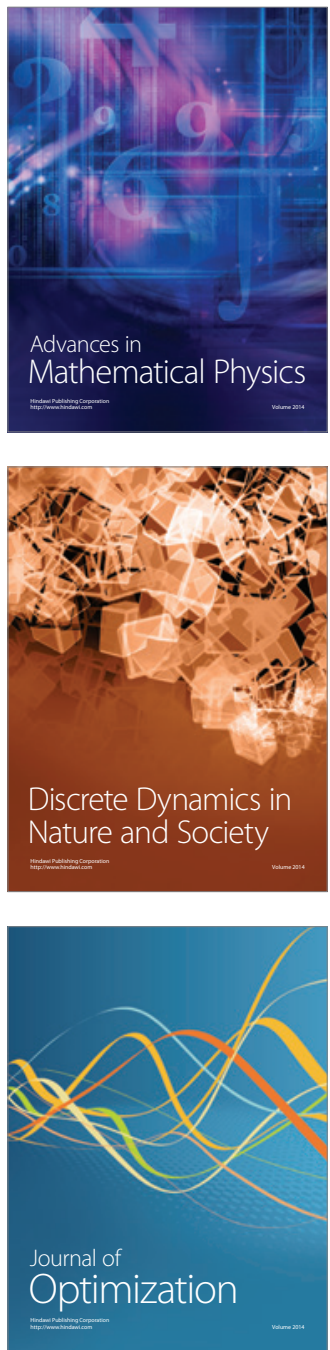